\title{
Analysis of a distinct speech disorder seen in chronic manganese toxicity following Ephedrone abuse.
}

\author{
video is part of $\mathrm{ms}$
}

\author{
M. Selikhova, MRCP, $\mathrm{PhD}^{2}$ E Tripoliti, PhD(4), L. Fedoryshyn, MD, PhD ${ }^{1}$, Y. Matvienko, \\ MD,PhD(5), H Stanetska, PhD ${ }^{1}$, M. Boychuk, MD ${ }^{1}$, I. Komnatska, MD, PhD ${ }^{3}$, A.J Lees, \\ MD, PhD, FRCP ${ }^{2}$ and Y. Sanotsky, MD,PhD ${ }^{1}$. \\ ${ }^{1}$ Department of Neurology, Lviv Regional Clinical Hospital, Ukraine \\ ${ }^{2}$ Institute of Neurology, Reta Lila Weston Institute of Neurological Studies, UCL, UK \\ 3 Department of MRI, Central Hospital of the Lviv Regional Railway, Lviv, Ukraine \\ 4 Unit of Functional Neurosurgery, Institute of Neurology, UCL London, United Kingdom \\ 5 Department of Neurology, Lviv National Medical University, Lviv, Ukraine
}

Running Title: Ephedrone induced dysarthria

Address for correspondence:

Dr Marianna Selikhova - a corresponding author

Reta Lila Weston Institute of Neurological Studies

1 Wakefield Street

London, WC1N 1PJ, United Kingdom.

Telephone: +44-20-7679 4246

Fax: $\quad+44-20-72784993$

Email: m.selikhova@talk21.com

Disclosure Statement. The authors have no conflict of interest that relates to research covered in this article.

\section{Acknowledgments}

Authors acknowledge Reta Lila Weston Institute of Neurological Studies for sponsoring Dr Selikhova's visit to Lviv, Ukraine for collecting research data. 


\begin{abstract}
Introduction: In the last fifteen years a new cause of chronic manganese toxicity has been recognized. It follows recreational intravenous injections of Ephedrone, synthesized from a cold remedies contained pseudoephedrine. Potassium permanganate is used as an oxidant. It presents with severe parkinsonism-dystonia and a characteristic dysarthria.
\end{abstract}

Objectives. We performed a focus perceptual study of dysarthria in Ephedrone induced parkinsonism and compared the findings with the speech disorders seen in Parkinson's disease (PD) and Progressive Supranuclear Palsy (PSP).

Methods. A digital voice recording, perceptual speech analysis (Darley, 1975), serial neurological assessment and Brain Magnetic Resonance (MR) imaging were performed at the Lviv regional Clinical Hospital. The results were analysed at the Institute of Neurology in London.

Results. Dysarthria developed after 8.5 \pm 3.2 months of daily intravenous Ephedrone abuse and was an initial symptom in a third of cases. It was characterised by a robotic-flat prosody, whispering or continuous phonation, an inability to regulate pitch and volume, frozen lip articulation, a variable degree of dystonic tightness, difficulties in speech initiation and palladia, There was no nasality and swallowing was normal. In some patients speech deteriorated even after the discontinuation of Ephedrone.

MR imaging, performed soon after drug cessation showed T1 signal hyperintesity in striatum and pallidum, especially in the Globus Pallidum interna. 
Conclusion. Ephedrone induced chronic manganese toxicity can lead to a mixed hypokineticdystonic dysarthria with a distinct dystonic pattern. Perceptual speech analysis can be a helpful ancillary investigation in the differential diagnosis of parkinsonism, and may permit the recognition of chronic manganese toxicity.

\section{Key words: Parkinsonism; Dysarthria, manganism; Globus Pallidum}

\section{Introduction}

Chronic manganese (Mn) poisoning is a recognised cause of basal ganglia toxicity[1-2]. A severe irreversible parkinsonism with dystonia was first reported by J. Couper (1837) in miners following inhalation of dioxide manganese in ore dust [3]. The characteristic clinical features include hypomimia, risus sardonicus incontinent laughter, early retropulsion with falls backwards and a dystonic cock gait [4]. Whispering dysarthria and mutism have been recorded [5]. As the fourth most widely used metal, manganese poisoning continues to be a common occupational hazard [6-7].T1 MR hyperintensities in the basal ganglia, typical of manganese accumulation, help the diagnosis, but may disappear after exposure has ceased [8,9]. Chronic liver disease is another cause of accumulation of manganese in the basal ganglia. Cirrhosis induced parkinsonism is reported in $1 \%$ patients with liver failure [10]. Parkinsonism with dystonia, polycythaemia and liver cirrhosis can develop due to mutation error in manganese transporter SLC30A10. This is a protective enzyme against manganese toxicity [11]. Over the last decade large numbers of cases of severe parkinsonism with dystonia have been recognized in Russia, Ukraine, the Baltic States and other parts of Eastern Europe following intravenous Ephedrone abuse. This amphetamine like substance was synthesized by kitchen chemists by 
mixing Coldact, a cold remedy containing pseudoephedrine with Potassium permanganate for oxidation[4,12,13]. The clinical and neuroimaging picture closely resembled previous reports of severe chronic manganese poisoning [14-15]. Dopamine transporter imaging was normal[14]. The disease continued to progress despite drug cessation in some cases and dysarthria became the most disabling complaint for many patients[12].

A quantitative acoustic analysis of Ephedrone induced dysarthria which was recently performed by Ruzs et al (16), revealed a distinct variant of mixed dysarthria with a combination of hyperkinetic and hypokinetic components representing the altered motor programming of dystonia and bradykinesia in Ephedrone-induced parkinsonism. We used perceptual speech analysis to evaluate further the pattern of speech disturbance in Ephedrone induced parkinsonism caused by chronic manganese toxicity and to compare it with the dysarthria seen in PD and Progressive Supranuclear Palsy (PSP)

\section{Materials and methods.}

Fourteen men (mean age 29.9), former Ephedrone addicts, who stopped injecting Ephedrone more than two years prior to speech assessment, were included in the speech study. All patients have been participating in the longitudinal UK-Ukraine study on Ephedrone induced dystonia with parkinsonism based at the Lviv Regional Clinical Hospital (Ukraine). A repeat neurological assessment, Mini-Mental examination (MMSE), video recording as well as repeat MR brain imaging were performed at the same time as speech assessment. Patient's baseline clinical and imaging data, published previously, were used for analysis of the disease course. Five patients had DAT scans previously, which were all normal [15].Unified PD rating scales (UPDRS) on the 
subjects was performed at the time of the speech evaluation by two experience neurologists (L.F. and M.S), with an expertise in Movement disorders [19]. Selective subscales of UPDRS are presented in the table 1 . In addition, Semi-quantitive scale was used for evaluation of oro-motor function and dystonia of the limbs. Speech was graded according UPDRS, item 18: 0 = Normal; 1 = Slight loss of expression, diction and/or volume; 2 = Monotone, slurred but understandable; moderately impaired; 3 = Marked impairment, difficult to understand; 4 = Unintelligible.

Evaluation of speech included digital voice recording and consisted of diadochokinetic rate, maximum sustained phonation with sustaining the vowel /a/ in one breath; repetition /pa/-/ta//ka/ syllable as fast and as slow as they can for $30 \mathrm{sec}$ each; a one-minute monologue and repetition of the sentence "buy Bobby a puppy". Speech recording was performed in a quiet room, using a head-mounted condenser microphone M-Audio MicroTrack 24/96 placed approximately $5 \mathrm{~cm}$ from the subject's mouth. A detailed perceptual analysis of articulation, respiration, resonance, phonation, prosody, rate of speech, using a scale first recommended by Darley and colleagues (1975) scale, was then performed by both Ukranian and UK Speech therapists [18]. Rating of 6 speech subsystems from 0 (no function) to 7 (normal function) with a total of 42 .

Written informed consent for the participation in research and video recording was provided by each patient.

MR brain scans were performed (0.5 Tesla, GE medical Systems) using a T1 weighted sequence (TR, 500 milliseconds; TE, 11 milliseconds) in the coronal, sagittal and axial planes and a T2 weighted sequence (TR, 4000 milliseconds; TE 80 milliseconds) in the axial plane; The T1 weighted images were inspected for the presence and severity $\mathrm{T} 1$ hyperintense signal change in 
the basal ganglia and brain stem nuclei in 14 anatomical structures. Evaluation of signal intensity was made by placing the region of interest (ROI) in areas with the highest intensity in the grey matter defined by visual comparison with subcortical frontal white matter. This approach replicated the standard method of evaluation pallidal index [9]. Semi-quantitive analysis with a 4 point rating scale and the signal intensity of normal frontal white matter (WM) as a reference was used.

MR data showed an abnormal T1 hyperintense signal in 11 of the 14 patients. Three patients who presented after 5 years of exposure, had normal MRI studies. The MRI findings were evaluated by the neuroradiologist without knowledge of the clinical findings.

Fifty four patients with Parkinson's disease (PD) from the National Hospital for Neurology and Neurosurgery, who were assessed on- and off medication before and after one year of deep brain stimulation surgery, served as control groups. Mean age of the patients with PD was 58.8 years ( \pm 6.3$)$, disease duration 12.5 years $( \pm 4.7)$, levodopa equivalent daily dose $1556( \pm 671)$ and UPDRS-III off-medication $48.1( \pm 17.9)$ and on-medication $12.4( \pm 7.8)$. A smaller group of 5 patients with probable PSP were also studied at the National Hospital. Their mean age was 69.9 $( \pm 9.12)$ years, disease duration- $5( \pm 3.3)$ years. By the time of speech assessment the patients had a prominent postural instability with the tendency to fall backwards, a marked supranuclear gaze palsy, gate freezing (3), symmetrical akinetic-rigid syndrome unresponsive to LD, progressive disease course, no dementia, no hallucinations, cerebellar syndrome, no cortical sensory deficit. The speech pattern of both patients in the control groups was assessed using the 
same perceptual scale (19) (Table 3). Statistics included two-tailed $t$-test or the Mann Whitney $U$ test, as appropriate, for continuous variables, using STATISTICA version 6.0.

\section{Results.}

The first neurological symptoms developed after 6-12 months (8.5 \pm 3.2 months) of daily IV Ephedrone abuse including progressive dysarthria, slowness of movements, retropulsion, falls backwards, particularly on turning, apathy and abulia. One patient presented with manganese madness (code 3). The disease progression was fast within the first few weeks, then slowed down. Dysarthria was the initial symptom in $31 \%$ and continued to deteriorate after 6 months in most cases. Dystonia and tremor appeared later, in 6-12 months after the cessation of Ephedrone. Progression of dysarthria, dystonia and postural instability continued for months and years after of the drug cessation. This was evident in cases with prolong disease duration for 5-7 years. An average UPDRS total score was $51.6 \pm 14.6$ compared to previous $45.8 \pm 13.7$ two years ago. The core neurological features in each patient are showen in table 1. All patients on the neurological examination had hypomimia with a constant dystonic grin, resembling a natural joyful expression, slow and jerky tongue movements, primitive reflexed of oral automatism and a forced involuntary laughter. Two patients had a mild hypersalivation and one- a mild difficulty with swallowing. Palatal movements were normal. Dysarthria was moderate to severe in eleven out of 14 patients according to UPDRS score, item 18. Only three patients had a mild speech impairment (table 1). Patients complained that their speech had become slurred, slow, laboured and often hard to initiate. Many reported a tight feeling in their throat, like a "lump", a loss of speech volume and an inability to raise their voice. Two patients were mute (code 1 and 3), which developed within two years after cessation of the drug. There was a complete loss of initiation, no speech volume, slow tongue movements and palilalia was identified during their occasional effortful production of single words (yes- no). 
Almost all patients had evident rigidity of axial muscles, whereas limbs rigidity was mild and belonged to lead-pipe type. Falls backwards and a staggering gait with dystonic posturing of the limbs and trunk, resembling "cock gait" were observed. Asymmetrical dystonic posturing of the feet, striatal toes, hands dystonia of "dinner folk" and pseudoparalisis patterns were evident. Apraxia of eyelid opening was seen in 9 patient. Ten patients had micrographia. Five patients had an assymmetrical actional tremor in their limbs. Rest tremor was rare. Severe dysarthria (score 3-4 on UPDRS- 18) was associated with more severe fine finger slowness, $2.5 \pm 0.5$ (UPDRS, item 23$)$, compared to $1.4 \pm 0.8$ on mild-moderate dysarthria $(\mathrm{p}=0.02)$. An average MMSE was $29( \pm 1.2)$.

Most patients (8) had parkinsonism with dystonia phenotype. An interview of motor and speech symptoms showed that all patients with dystonic voice patterns had dystonia in the limbs, trunk and face (tables 1). Three patients with hypokinetic speech, without signs of dystonia presented with predominant parkinsonism phenotype with little or none limb dystonia. This observation is in keeping with the report of Rusz et al (2014), who showed a significant relationship between several acoustic speech dimensions and the motor ratings of bradykinesia and dystonia [16]. In particularly, the dystonia severity correlated with speech timing parameters. Thus, the speech pattern was largely corresponding to the motor phenotype of Ephedrone induced parkinsonism.

\section{Levodopa test was performed on baseline assessment with Madopar dispersible 50/200 mg and} showed less than $30 \%$ response. The following LD trial did not show improvement: three discontinued Madopar therapy at doses between 300 and $500 \mathrm{mg} /$ day because of intolerable nausea, 2 stopped taking $650 \mathrm{mg} /$ day Madopar after 2 months without clinical benefit, and a further 2 patients were able to tolerate $750 \mathrm{mg}$ a day for 3 months but failed to derive benefit. 


\section{$\underline{\text { Perceptual analysis of Ephedronic dysarthria }}$}

Detailed results from perceptual analysis on each patient presented in the table 2.

Phonation: All but one patients had a markedly reduced speech volume. Seven had a whispering pattern, including two patients with a complete aphonia. One patient had normal volume but with a continuous monotone voice and a complete inability to change pitch and loudness (monoloud) Five patients also had a strained-strangled dystonic voice quality.

Prosody: Speech was monotonous in all patients, with lack of intonation and emphasis. Five patients had so restricted speech prosody that could be classified as robotic.

Articulation:_The main articulatory feature affected was the range of movement with minimum amount of lip and jaw movement (frozen upper lip). On non-speech oral mechanism examination there was also evidence of tongue rigidity and jerky movements (table 1). The sounds were prolonged and with equal stress. Palilalia was present in eight patients.

Respiration: Six cases had difficulty completing long sentences. Eight patients showed some difficulty with reduced breath support. Such difficulties could contribute to reduced physiologic support for speech and some of the overall phonatory and prosodic abnormalities

Nasality: There was no perceptually prominent nasality.

Rate: Rate was variably affected, either slow $(\mathrm{N}=7)$ with prolonged vowels or fast, festinating speech. $(\mathrm{N}=6)$. Delay in initiation was observed in six.

\section{Comparison with parkinsonian dysarthria}


The comparison of the average perceptual speech scores of patients with Ephedrone induced parkinsonism and patients with PD off- and on- medication and PD patients post bilateral Subthalamic nucleus- deep brain simulation (STN-DBS) are shown in table 2. Despite the fact that Ephedronic dysarthria can be broadly classified as hypokinetic, its severity is significantly greater than the dysarthria of patients with PD-medical group in all aspects of perceptual analysis with the exception of phonation (table 3). The main areas of difference are prosodic impairment and articulation. The degree of monotonicity in Ephedronic dysarthria was much greater and the quality of voice was more robotic, due to the severe lack of stress and the continuous voicing. Articulation was also differently affected: in PD there was a lack of precision possibly due to spirantisation (i.e. articulatory undershooting, the replacement of stop with a fricative), whereas in Ephedronic dysarthria the main problem was that of reduced range of movements for lip and tongue muscles. As can be seen from the table 3, the disturbance of articulation, respiration, phonation and prosody in Ephedronic dysarthria resemble in pattern and severity of the dysarthria in cases of Parkinson's disease after bilateral STN-DBS, with the exception of nasality [19].

\section{Comparison with PSP dysarthria}

Speech in PSP patients was different from PD cases and characterized by monopitch with hoarseness, hypernasality, imprecise articulation and a slow rate. In comparison with the Ephedronic patients, the PSP group exhibited worse respiratory control for speech, a hoarse weak voice, imprecise articulation, and slower rate of speech and occasional hypernasality. Characteristics, more often presented in Ephedronic dysarthria, rather than PSP, include strainedtight voice, monotone-monoloud speech, and variable speech rate. Similarities include the 
hypokinetic components, occasional presence of palilalia and the possibility of mutism. Both patient groups have shown difficulty with raising vocal volume on command and thus in making gains through behavioural therapy. These distinctions suggest that there is a different pathophysiology of speech in PSP than in PD patients.

MR brain image in Ephedrone induced parkinsonism -dystonia.

\section{MRI findings are presented in Table 4. Patients with abnormal MRI all had symmetrical T1}

hyperintense signal in the Globus Pallidus (GP), the most severe in its medial part with an average score of 2.36 , followed by the lateral pallidum (average score:1.82). Other frequently involved structures were the subthalamic nucleus and substantia nigra reticulate ( $\mathrm{SNr}$ ), showing hyperintensity in 6 and 7 patients, respectively. (fig. 1) The putamen showed lateral hyperintensity in seven patients. Involvement of posterior fossa structures was seen in less than half patients. The MRI studies performed closer to the time of drug exposure, showed more intense and more widespread T1 hyperintense signal changes. In the patients with severe neurological deficit MR signal change in the pallidum was still visible 3-5 years after the last exposure to ephedrone. MRI studies performed after 5 years of drug cessation were reported as essentially normal. (fig.2) The reversibitly of MR signal change has previously also been documented in patients with liver failure after liver transplantation [9].

\section{Discussion}

Rodier (1955) first reported on severe speech disorder in chronic manganism: "Neurological symptoms usually began with monotonous slow slurred speech with acquired stuttering, and muteness eventually ensues"[20]. Clinical and radiological features of Ephedrone induced parkinsonism are closely resemble previously described cases of severe manganism in miners. 
This allowed us to observe and study more closely the pattern of speech. Levin (2005) described speech in Ephedronic encephalopathy as slow, hoarse, quiet, effortful and difficult to initiate. Three out of his 21 patients became mute and $14 \%$ had ataxic component of speech [21]. We performed a comparison of Ephedronic dysarthria with hypokinetic dysarthria in PD and PSP using perceptual analysis (Darley 1975). Dysarthria in ex-Ephedrone users was an initial symptom in a third cases. Ephedronic speech was hypokinetic and monotonous, with inappropriately slow or accelerated speech rate. But they were unable to change pitch and loudness. 50\% had whispering dysarthria. Another distinguishing feature was a profound lack of prosody and lip articulation. Mutism is not a feature in PD, but was seen in Ephedrone induced disarthria (14\%). Frequent palilalia (57\%) and difficulties in initiation speech (43\%) point towards the pallidal damage. By contrast to PD patients, over 74\% ex -Ephedrone addict cases had a dystonic component resulted in prolonged and strained hypokinetic speech.

Dysarthria of mixed type is typical for Parkinson plus syndromes. In PSP hypokinetic, spastic and ataxic types are consistently identified in various combinations, depending on the loci of the neuropathologic changes. Slurred delayed speech with raspy or hoarse voice with a spastic component is often reported [22]. Dysarthria in our patients with PSP was associated with monopitch, hoarseness, nasal emission, hypernasality, imprecise articulation, slow rate, and a prominent mixed hypokinetic with spastic features. Similarly to PSP, Patients with Ephedrone induced parkinsonism were unable to increase the voice volume. However, in contrast to PSP, nasality was not a feature and there were whispering but otherwise intelligible consonants. Also, swallowing was preserved even in most severe dysarthria cases by contrast to PSP. The absence of ataxic and nasal components distinguished Ephedrone dysarthia from the speech in MSA [23] Ephedrone induced dysarthria has perceptual similarity with post STN-DBS speech in PD 
patients. Astrom et al (2010) investigated the spread of the electrical current in post bilateral STN-DBS patients and found that patients with bilaterally medially placed electrodes (in the pallidothalamic tract) had more severely affected speech [24].

Results of MRI scans which was done soon after Ephedrone exposure, showed T1 hyperintense signal change suggestive of bilateral striatal accumulation of manganese, predominantly in medial part of GP and to less degree in lateral putamen, SN reticulate and STN, similarly to imaging in chronic manganese poisoning [25]. Degeneration of nerve cells in pallidum, especially in the medial segment with a prominent demyelination and moderate astrocytic proliferation has been reported in chronic manganism. [26] An exclusive role of GP in control of initiation of gait and speech was illustrated by Feve et al (1993), who presented four cases with pure circumscribed bilateral lesions of the Globus pallidum after a hypoxic-ischemic event [27]. The clinical picture consisted of speech initiating difficulties with hypophonia and pallilalia-mutism and a marked axial motor impairment but minimal distal rigidity, profound loss of postural reflexes, akinesia and gait freezing.

The voice loudness and speech initiation was closely associated with involuntary laughter and abulia in Ephedrone induced parkinsonism. This was first reported by Levin (2005) who also noted a beneficial effect of SSRI on dysarthria [21] This observation is supported by the experimental data on vocalization in mammals, which found that the readiness of vocal expression is controlled by the limbic vocal pathway from anterior Cingulate cortex to phonatory motoneurons [29]. Akinetic rigid mutism due to bilateral lesions of the globus pallidus interna with ventral extension, disrupting the anterior cingulate frontal-subcortical circuit, has been reported [28].Functional neuroimaging would help to clarify the altered basal ganglia circuits, 
responsible for manganese induced dysarthria . Hypophonic component of dysarthria showed some improvement with systematic speech therapy. Dystonic component remained unchanged or even worsened in our cases, similarly to the results from speech therapy in patients post bilateral STN-DBS[30]. Ephedronic dysarthria remains a debilitating syndrome requiring further investigation for its nature and symptoms management.

\section{Word cound : 3,046}

\section{Reference}

1. Huang C-C, Chu N-S, Lu C-S, et al. Long-term progression in chronic manganism: ten years of followup. Neurology 1998;50: 698-700.

2. Racette BA, Antenor JA, McGee-Minnich L et al . [18F]FDOPA PET and Clinical Features in Parkinsonism Due to Manganism. Mov Disord 2005;20: 492-496. DOI: 10.1002/mds.20381

3. Couper $\mathbf{J}$.On the effects of black oxide of manganese when inhaled into the lungs. Br Ann Med Pharmacol 1987; 1: 41-42.

4. Sanotsky Y, Lesuk R, Fedorishin L, Komnatska I, Matvienko Y and Fahn S. "Manganese Encephalopathy due to Ephedrone abuse. Mov disord 2007; 22:1337-1343

DOI: 10.1002/mds.21378Calne DB,Chu NS,Huang CC at al .Manganese and parkinsonism: similarities and differencies. Neurology 1994; 44: 262 PMID:7936278

5. Edsall DL, Wilbur FP, Drinker CK..The occurrence, course and prevention of chronic manganese poisoning. J Ind Hyg 1919; 1:183- 193

6. Jankovic J. Searching for a relationship between manganese and welding and Parkinson's disease. Neurology 2005;64:2021-8. doi: 10.1212/01.WNL.0000166916.40902.63

7. Cowan DM, Fan Q, Zou Y et al. Manganese exposure among smelting workers: blood manganese-iron ratio as a novel tool for manganese exposure assessment. Biomarkers. 2009 Feb;14(1):3-16. doi: 10.1080/13547500902730672.

8. Hernandez EH, Discalzi G, Valentini C et al. Follow-up of patients affected by manganese-induced Parkinsonism after treatment with CaNa2EDTA. NeuroToxicology 2006; 27, 3: 333-339. doi:10.1016/j.neuro.2005.09.003

9. Naegele T, Lauchart W, Gregor M et al. MR imaging and (1) H spectroscopy of brain metabolites in hepatic encephalopathy: time -course of renormalization after liver transplantation.Radiology 2000; 216:683-91 
10. Klos KJ, Ahlskog JE, Josephs KA et al. Neurologic spectrum of chronic liver failure and basal ganglia T1 hyperintensity on magnetic resonance imaging: probable manganese neurotoxicity. Arch Neurol 2005;62(9):1385-90 doi:10.1001/archneur.62.9.1385.

11. Tuschl K, Clayton PT, Gospe SM et al. Syndrome of hepatic cirrhosis, dystonia, polycythemia, and hypermanganesemia caused by mutations in SLC30A10, a manganese transporter in man. Am J Hum Genet. 2012 Mar 9;90(3):457-66.

12. Levin O, Fedorova N, Amosova N, Schtok V. Ephedronic Parkinsonism. Neurological Journal 2000;2: 8-14

13. Bie R, Gladstone R, Strafella A, Ko Ji-Hyun, Lang AE. Manganese-induced Parkinsonism associated with Methcathinone (Ephedrone) abuse. Arch Neurol 2007;64: 886-889 doi:10.1001/archneur.64.6.886

14. Selikhova M, Fedoryshyn L, Matviyenko Y et al.Parkinsonism and dystonia caused by the illicit use of ephedrone-a longitudinal study. Movement Disorders 2008; 23(15): 2224-2231.

DOI: $10.1002 / \mathrm{mds} .22290$

15. Sikk K, Taba P, Haldre $\mathrm{S}$ et al. Clinical, neuroimaging and neurophysiological features in addicts with manganese-ephedrone exposure Acta Neurologica Scandinavica 2010; 121( 4): 237-243.

DOI: 10.1111/j.1600-0404.2009.01189.x

16. Rusz J, Megrelishvili M, Bonnet $\mathrm{C}$ et al. A distinct variant of mixed dysarthria reflects parkinsonism and dystonia due to ephedrone abuse. J Neural Transm 2014; 121(6):655-64. doi: 10.1007/s00702-0141158-6.

17. Fahn S, Elton R. Unified Parkinson's disease rating scale. Macmillan healthcare Information 1987;5: 153-163

18. Darley FL, Aronson AE and Brown JR (1975) Motor speech disorders. Philadelphia: W.B. Sanders Duffy J (2005) Motor speech disorders: Substrates, Differential diagnosis and management. Elsevier Mosby: 175 -179 doi: 10.1044/1092-4388(2012/11-0309)

19. Tripoliti E, Zrinzo L, Martinez-Torres I et al. Effects of Contact Location and Voltage Amplitude on Speech and Movement in Bilateral Subthalamic Nucleus Deep Brain Stimulation. Mov Dis 2008. 23(16): 2377-2383.doi:10.1007/978-3-642-03889-1_89

20. Rodier J. Brit. J. industr. Med 1955. 12, 21

21. Levin O. Ephedrone Encephalopathy. J Neurology and Psychiatry S.S.Korsakoff 2005; 7: 12-20

22. Kluin K.J, Gilman S, Norman L et al. Neuropathological Correlates of Dysarthria in Progressive Supranuclear Palsy. Arch Neurol 2001; 58:265-269. DOI: 10.4103/0028-3886.41987

23. Huh YE, Park J, Suh MK. et al. Difference in early speech patterns between Parkinson variant of mulpiple system atrophy and Parkinson's disease. Brain\&Language 2015; 147:14-20.

Doi:10.1016/j.bandl.2015.04.007 
24. Astrom M, Tripoliti E, Hariz MI et al.Patient-specific model-based investigation of speech intelligibility and movement during deep brain stimulation. Stereotact Funct Neurosurg 2010;88(4):224-33. doi: 10.1159/000314357

25. Park J, Kim Y, Kim JW. High signal intensities on T1-weighted MRI in the spectrum of manganese symptomatology. Webster LR (2007) Neurotoxicity syndrome. New York : Nova Biomedical Books: 249-260.

26. Maeda H,Sato M, Yoshikawa A et al. Brain MR imaging in patients with hepatic cirrhosis: Relationship between high intensity signal in basal ganglia on T1-weighted images and elemental concentrations in brain. Neuroradiology 1997;39:546-50

27. Feve AP, Fenelon G, Wallays C et al. Axial Motor Disturbances After Hypoxic Lesions of the Globus Pallidus. Movement Disorders 1993;8( 3): 321-326 DOI: 10.1002/mds.870080311

28. Jürgens $\mathrm{U}$. The squirrel monkey as an experimental model in the study of cerebral organization of emotional vocal utterances. Eur Arch Psychiatry Neurol Sci. 1986;236(1):40-3.

29. Mega MS, Cohenour RC. Akinetic mutism: disconnection of frontal-subcortical circuits. Neuropsychiatry Neuropsychol Behav Neurol. 1997 Oct;10(4):254-9.

30. Tripoliti E, Strong L, Hickey F, Foltynie T, Zrinzo L, CandelarioJ, Hariz MI, Limousin P. Treatment of dysarthria following subthalamic nucleus deep brain stimulation for Parkinson's Disease. Mov Disord 2011; 26(13):2434-6. doi: 10.1002/mds.23887

\section{Ethical standards}

This study was approved by the local ethical committee and have therefore beend performed in accordance with the ethical standards laid down in the 1964 Declaration of Helsinki and its later amendments. Each patient gave their informal consent prior to the inclusion in the study. 
Table 1: Motor speech control in Ephedrone induced Parkinsonism.

\begin{tabular}{|c|c|c|c|c|c|c|c|c|c|c|c|c|c|c|}
\hline $\begin{array}{l}\text { Patient } \\
\text { number }\end{array}$ & 1 & 2 & 3 & 4 & 5 & 6 & 7 & 8 & 9 & 10 & 11 & 12 & 13 & 14 \\
\hline $\begin{array}{l}\text { Speech } \\
\text { UPDRS-18 }\end{array}$ & 4 & 4 & 4 & 4 & 3 & 2 & 2 & 2 & 1 & 1 & 2 & 3 & 1 & 2 \\
\hline $\begin{array}{l}\text { Signs } \\
\text { duration, yrs }\end{array}$ & 5.5 & 4 & 2.5 & 5 & 4 & 5.3 & 4.5 & 2.5 & 7 & 2 & 2 & 3.5 & 4.5 & 7 \\
\hline Dystonic smile & + & + & + & + & +++ & + & + & + & + & + & + & ++ & + & + \\
\hline $\begin{array}{l}\text { Frozen upper } \\
\text { lip }\end{array}$ & +++ & +++ & +++ & +++ & +++ & ++ & ++ & +++ & ++ & ++ & ++ & ++ & + & $\begin{array}{l}+ \\
+\end{array}$ \\
\hline $\begin{array}{l}\text { Tongue } \\
\text { movement }\end{array}$ & +++ & +++ & +++ & +++ & +++ & ++ & + & +++ & + & 0 & + & ++ & + & + \\
\hline micrography & +++ & + & 0 & + & + & + & 0 & 0 & + & 0 & +++ & + & + & ++ \\
\hline $\begin{array}{l}\text { Limb } \\
\text { dystonia* }\end{array}$ & 0 & + & ++ & ++ & ++ & 0 & ++ & + & + & ++ & 0 & 0 & + & + \\
\hline $\begin{array}{l}\text { apraxia eyelid } \\
\text { opening }\end{array}$ & + & + & + & + & +++ & 0 & 0 & + & + & + & 0 & 0 & + & 0 \\
\hline Tremor at rest & 0 & 0 & + & + & + & 0 & + & 0 & 0 & 0 & 0 & 0 & + & + \\
\hline $\begin{array}{l}\text { Pull test } \\
\text { UPDRS } 30\end{array}$ & 2 & 3 & 3 & 3 & 3 & 2 & 3 & 3 & 2 & 2 & 2 & 2 & 1 & 1 \\
\hline $\begin{array}{l}\text { Axial rigidity } \\
\text { UPDRS } 22\end{array}$ & 3 & 3 & 3 & 2 & 2 & 1 & 2 & 1 & 1 & 2 & 3 & 2 & 1 & 2 \\
\hline $\begin{array}{l}\text { Finger tap } \\
\text { UPDRS } 23\end{array}$ & 3 & 3 & 3 & 3 & 3 & 2 & 1 & 3 & 1 & 2 & 2 & 1 & 1 & 2 \\
\hline
\end{tabular}

Grade of symptoms: + mild; ++ moderate; +++ severe presentation; 0- absent.

UPRDS subscales original version is used. *this score reflects the most affected limb. 
Table 2. Speech perceptual analysis of Ephedrone induced dysarthria.

\begin{tabular}{|c|l|l|l|l|l|l|l|l|l|l|l|l|l|l|}
\hline $\begin{array}{c}\text { Patient' code } \\
\begin{array}{c}\text { Darley score: } \\
\text { total }\end{array}\end{array}$ & $\mathbf{1}$ & $\mathbf{2}$ & $\mathbf{3}$ & $\mathbf{4}$ & $\mathbf{5}$ & $\mathbf{6}$ & $\mathbf{7}$ & $\mathbf{8}$ & $\mathbf{9}$ & $\mathbf{1 0}$ & $\mathbf{1 1}$ & $\mathbf{1 2}$ & $\mathbf{1 3}$ & $\mathbf{1 4}$ \\
\hline - Articulation & 4 & 3 & 3 & 4 & 4 & 4 & 5 & 5 & 6 & 5 & 5 & 5 & 7 & 4 \\
\hline - Respiration & 5 & 7 & 4 & 5 & 4 & 5 & 6 & 6 & 7 & 6 & 7 & 5 & 7 & 7 \\
\hline- Nasality & 7 & 7 & 5 & 7 & 5 & 5 & 6 & 7 & 7 & 7 & 7 & 7 & 7 & 7 \\
\hline - Phonation & 1 & 2 & 1 & 1 & 5 & 4 & 5 & 5 & 6 & 6 & 5 & 6 & 6 & 4 \\
\hline - Prosody & 2 & 1 & 1 & 1 & 4 & 4 & 3 & 4 & 4 & 4 & 4 & 3 & 4 & 4 \\
\hline - Rate & 4 & 1 & 1 & 4 & 3 & 3 & 3 & 4 & 4 & 4 & 4 & 3 & 7 & 5 \\
\hline Weak voice & + & + & + & + & + & + & + & + & 0 & + & + & 0 & 0 & + \\
\hline Monotone & + & + & + & + & + & + & + & + & + & + & + & + & + & + \\
\hline Monolaud & + & + & + & + & + & + & + & + & + & + & + & + & + & + \\
\hline Rate Slow/fast & fast & slow & slow & slow & slow & fast & slow & fast & fast & slow & fast & slow & 0 & fast \\
\hline Initial delay & + & + & + & + & + & 0 & 0 & 0 & 0 & 0 & 0 & 0 & + & 0 \\
\hline Palilalia & + & + & + & + & 0 & 0 & 0 & 0 & + & + & 0 & + & 0 & + \\
\hline $\begin{array}{c}\text { Voicing pattern } \\
\text {-hypokinetic }\end{array}$ & + & + & + & + & + & + & + & + & + & + & + & & + & + \\
\hline Tight/dystonic & + & + & + & + & + & + & + & + & + & + & & + & \\
\hline- Robotic & + & + & + & + & & & + & & & & & + & \\
\hline - Contineous & mute & + & mute & & + & & + & & & & & + & & \\
\hline - Whispering & + & + & + & + & & + & & & & & + & & + \\
\hline
\end{tabular}

Darley (1975) scale (18) rating from 0 (no function) to 7 (normal function), 42 total.

Symptoms present: +; 0- absent. 
Table 3 Comparison of dysarthria between Ephedronic Parkinsonism $(\mathrm{N}=14)$ and Parkinson's Disease $(\mathrm{N}=54)$

\begin{tabular}{|l|l|l|l|l|l|l|l|}
\hline $\begin{array}{l}\text { Darley } \\
\text { scale } \\
\mathrm{M} \pm \text { SD }\end{array}$ & $\begin{array}{l}\text { Ephedronic } \\
\text { parkinsonism }\end{array}$ & $\begin{array}{l}\text { PD "off" } \\
\text { treatment }\end{array}$ & P 1-2 & PD "On" & P 1-3 & PD post & P 1-4 \\
\hline groups & \multicolumn{1}{|c|}{$(1)$} & $(2)$ & & $(3)$ & & $(4)$ & \\
\hline Articulation & $4.57 \pm 1.09$ & $5.9 \pm 0.8$ & 0,0002 & $5.6 \pm 1.3$ & 0,03 & $4.4 \pm 1.9$ & 0,3 \\
Respiration & $5.78 \pm 1.12$ & $5.0 \pm 1.0$ & 0,05 & $4.9 \pm 1.1$ & 0,04 & $4.4 \pm 1.9$ & 0,02 \\
Resonance & $6.5 \pm 0.85$ & $5.6 \pm 1.0$ & 0,01 & $5.6 \pm 0.8$ & 0,007 & $4.9 \pm 1.6$ & 0,001 \\
Phonation & $4.07 \pm 1.98$ & $4.8 \pm 1.0$ & 0,1 & $5.1 \pm 1.0$ & 0,09 & $4.4 \pm 1.5$ & 0,3 \\
Prosody & $3.07 \pm 1.27$ & $4.9 \pm 1.5$ & 0,001 & $5.3 \pm 1.4$ & 0,0001 & $4.1 \pm 1.8$ & 0,09 \\
$\begin{array}{l}\text { Rate } \\
\text { control }\end{array}$ & $3.57 \pm 1.50$ & $6.0 \pm 0.9$ & 0,001 & $5.8 \pm 1.3$ & 0,0001 & $5.2 \pm 1.6$ & 0,01 \\
Total score & $27.6 \pm 6.1$ & $32.4 \pm 4.6$ & 0,02 & $32.5 \pm 5.2$ & 0,02 & $27.3 \pm 8.8$ & 0,3 \\
\hline
\end{tabular}

Scale by Darley et al (1975) (18) with rating from 0 (no function) to 7 (normal function), total out of 42 . The table show the comparison of the average perceptual scores in patients with ephedronic parkinsonism (1) with PD patients, who were assessed off- medication (2), on- medication (3) and then after Bilateral STN-DBS (4).It showed a significant difference (P-value) in all parameters between 1-2 groups, wherease there was no difference in 4 parameters in 1-4 comparison.

Table 3. MRI findings in Ephedronic Encephalopathy

\begin{tabular}{|l|c|c|c|c|c|c|c|r|r|r|r|}
\hline patient's code & $\mathbf{1}$ & $\mathbf{3}$ & $\mathbf{4}$ & $\mathbf{5}$ & $\mathbf{7}$ & $\mathbf{8}$ & $\mathbf{9}$ & $\mathbf{1 0}$ & $\mathbf{1 1}$ & $\mathbf{1 2}$ & $\mathbf{1 3}$ \\
\hline years disease to MRI & 2.5 & 0.4 & 5 & 1 & 3 & 0.4 & 1 & 0.4 & 0.5 & 0.5 & 1.5 \\
\hline n.caudate head & 0 & 0 & 0 & 0 & 0 & 0 & 0 & 0 & 1 & 0 & 0 \\
\hline n.caudate rim & 0 & 0 & 0 & 0 & 0 & 0 & 0 & 0 & 2 & 0 & 0 \\
\hline n.caudate body & 0 & 0 & 0 & 0 & 0 & 2 & 0 & 0 & 1 & 0 & 0 \\
\hline gl.pallid med & 3 & 3 & 2 & 3 & 1 & 2 & 3 & 2 & 3 & 3 & 1 \\
\hline gl.pallidum lat & 3 & 2 & 1 & 2 & 1 & 1 & 2 & 2 & 3 & 2 & 1 \\
\hline Putamen & 0 & 1 & 0 & 1 & 1 & 2 & 0 & 1 & 2 & 1 & 0 \\
\hline Putamen lat rim & 1 & 2 & 0 & 2 & 0 & 0 & 0 & 2 & 3 & 0 & 0 \\
\hline Thalamus & 0 & 0 & 0 & 0 & 0 & 0 & 0 & 0 & 0 & 0 & 0 \\
\hline STN & 2 & 2 & 0 & 0 & 0 & 2 & 0 & 2 & 2 & 2 & 0 \\
\hline SN & 1 & 1 & 0 & 2 & 0 & 2 & 0 & 1 & 2 & 2 & 0 \\
\hline sup cerebellar ped & 0 & 1 & 0 & 2 & 0 & 2 & 0 & 0 & 2 & 0 & 0 \\
\hline n.dentatus Left & 0 & 0 & 0 & 1 & 0 & 0 & 0 & 2 & 0 & 0 & 0 \\
\hline n.dentatus R & 0 & 0 & 0 & 0 & 0 & 0 & 0 & 1 & 0 & 0 & 0 \\
\hline Pons rostral & 0 & 0 & 0 & 2 & 0 & 2 & 0 & 2 & 2 & 0 & 0 \\
\hline
\end{tabular}

The table lists the visual score of pathological signal increase on T1 weighted images in 14 anatomical structures. Grading for gray matter structures: 0, normal appearance; 1 - isointense to WM; 2- hyperintense to WM; 3- markedly 
hyperintense to WM. Grading for white matter: 0- normal; 1- hyperintense to WM; 2- markedly hyperintense to WM.

Fig. 1 MR brain image in Ephedrone induced parkinsonism.

Fig. 1 Two Non-enhanced coronal T1 weighted MR images showing bilateral markedly hyperintense signal in the globus pallidus, most severe in its medial part and, to a lesser degree in the substantia nigra reticulate, subthalamic nuclei, putamen and caudate nucleus. The Picture below: Normal repeat MR brain image in Ephedrone induced parkinsonism after 5 years without exposure.

Video Legend "Ephedronic dysarthria"

Segment 1 Patient 12. 26 y.o., with Ephedrone induced parkinsonism . 5.5 years of drug cessation . Continueous , tight robotic speech. No significant hypokinetic features.

\section{Segment 2}

Patient 7. 33 y.o., severe Ephedrone induced parkinsonism with dystonia. 8.5 years of drug cessation . Hypokinetic, continueous, tight robotic speech.

\section{Segment 3}

Patient 2. 33 y.o., severe Ephedrone induced parkinsonism with dystonia. Hypokinetic, whispering, continueous, tight robotic speech.

All authors have made a significant contribution to the conception, design, execution, or interpretation of the reported study. all co-authors have seen and approved the final version of the paper and have agreed to its submission for publication.

M. Selikhova (2) acquisition of data, design of the research, analysis and

interpretation of data, drafting of all the parts of the paper;

E. Tripoliti (2) - design of the reseach, analysing and interpreting data, drafting the methods and results parts of the paper,

L. Fedoryshyn (1) - acquisition of data, neurological assessments, technical and statistical expertise; evaluation of treatment effect; 
Y. Matviyenko (1) - conception of the research, analysis and interpretation of data

H Stanetska (1) - analysing and interpreting data, conception of the research

I. Komnatska (3) - organizing, performance and reporting of MRIs;

M. Boychuk (1) - acquisition of data, administrative support;

A. Lees (2) - conception of the research, interpretation of the data, critical revision of all the parts of the paper for important content, administrative support, obtaining funding, supervision;

Y. Sanotsky (1) - acquisition of data, conception and design of the research, Administrative and technical support, supervision, critical revision of all the parts of the paper for important content. 